\title{
Gastroprotective effect of cirsilineol against hydrochloric acid/ ethanol-induced gastric ulcer in rats
}

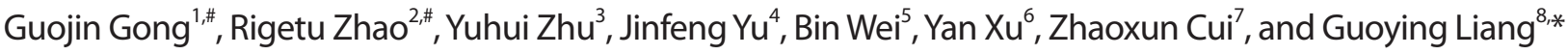 \\ 'Department of Gastrointestinal Surgery, Xichang People's Hospital, Xichang 615000, ${ }^{2}$ Department of Gastroenterology, The Affiliated Hospital of Inner \\ Mongolia University for Nationalities, Tongliao 028000, ${ }^{3}$ Department of Pharmacy, The Second Hospital of Jilin University, Changchun $130041,{ }^{4} \mathrm{General} \mathrm{Medi-}$ \\ cine Yantaishan Hospital, Yantai 264001, ${ }^{5}$ The People's Hospital of Guangxi Zhuang Autonomous Region, Nanning 530021, ${ }^{6}$ Chongqing University Affiliated \\ Tumor Hospital/Chongqing Cancer Institute, Chongqing 400030, ${ }^{7}$ Department of Oncology, Shaanxi Forest Industry Hospital, Xi'an 710000, ${ }^{8}$ Department of \\ Liver, Spleen and Stomach Diseases, First Affiliated Hospital of Heilongjiang University of Traditional Chinese Medicine, Heilongjiang 150040, China
}

\section{ARTICLE INFO}

Received January 5, 2021

Revised June 8, 2021

Accepted June 8, 2021

*Correspondence

Guoying Liang

E-mail: Igy1976190606@sina.com

Key Words

Antioxidant

Inflammation

Phenolic

Stomach ulcer

\#These authors contributed equally to this work.

\begin{abstract}
This study was designed to evaluate the gastroprotective activity of cirsilineol in hydrochloric acid $(\mathrm{HCl})$ /ethanol-induced gastric ulcer model. Cirsilineol was administered at the doses of 20 and $40 \mathrm{mg} / \mathrm{kg}$ in HCl/ethanol-induced rats. The gastroprotective ability was verified by determining the ulcer score, total acidity, hemoglobin, inflammatory cytokines, lipid peroxides, and enzymatic antioxidants superoxide dismutase (SOD) and catalase (CAT) in gastric tissue and serum biochemical analysis. The results showed a favorable increase in the hemoglobin level, antioxidant enzymes (SOD and CAT), restored electrochemical balance (carbon dioxide \& anion gap) while a noticeable decrease in ulcer index, total acidity, lipid peroxides, inflammatory cytokines (interleukin-1 beta [IL-1 $\beta]$, IL-6, and tumor necrosis factor alpha) in rats treated with the cirsilineol. The serum biochemical analysis on liver markers (alkaline phosphatases, alanine aminotransferase, and aspartate aminotransferase), kidney markers (urea, creatinine, albumin, globulin, total protein), and lipid profile (triglyceride, high-density lipoprotein, total cholesterol) were attenuated by cirsilineol treatment in rats. Histopathology showed enhanced gastric protection and preserved the integrity of gastric mucosa upon cirsilineol administration. These results ultimately suggest that cirsilineol has gastroprotective effects that prevent the development of gastric ulcer.
\end{abstract}

\section{INTRODUCTION}

Gastric ulcers are lesions in the mucosal lining of the digestive (gastrointestinal) system. The disease is characterized typically based on the anatomical location which is immersed in acid and pepsin [1]. Based on site of attack, gastric ulcer is recognized as the most prevalent chronic diseases of the peptic ulcer. A gastric ulcer occurs as a result of extended starvation, disruption in the barrier of gastric mucosa due to stress, nutrient deficiency, decreases in mucus secretion associated with prolonged or overdose ingestion of non-steroidal anti-inflammatory drugs (NSAID), smoking habit and infection with Helicobacter pylori [2]. Though gastric ulcer is a non-fatal disease, the symptom manifestation elicits discomfort, disturbs the daily routines and triggers mental distress in patient.

In the past decades, gastric ulcer affected a concerning number of people globally. Studies have revealed that the risk for developing a gastric ulcer is expected to be $10 \%$ approximately and the incidence peaks more than $80 \%$ by the age of 50 and above [3]. Though overall improvisation in hygiene and healthcare quality has influenced the declining rate in peptic ulcer especially in gastric ulcers, the amplified usage of NSAIDs has caused an increase (i) $\$$ This is an Open Access article distributed under the terms of the Creative Commons Attribution Non-Commercial License, which permits unrestricted non-commercial use, distribution, and reproduction in any medium, provided the original work is properly cited. Copyright @ Korean J Physiol Pharmacol, pISSN 1226-4512, eISSN 2093-3827
Author contributions: G.G. and R.Z. equal contribution (Manuscript writing, Animal treatment, Biochemical assays); Y.Z. (Biochemical assays-liver markers), J.Y. (Biochemical assays-kidney markers), B.W. (Histopathology), Z.C. (ELISA assays), Y.X. (Statistical analysis), G.L. (Experimental design, manuscript editing). 
in NSAIDs induced gastric ulcers. The complications of gastric ulcer impose significant morbidity and economic load on the health system and the society. According to literature, the total costs of gastric ulcer management have been expected to reach at least seven billion annually $[4,5]$.

Therapeutic management of gastric ulcers continues to be developed, with the aim of pain relieving, ulcer restoration and halting reoccurrence. Hence, the current pharmacological treatment is based on the inhibition of gastric acid secretion or protonpump inhibitors. Such derivatives are typically used to manage gastric ulcer clinically but in the long-term, they can't be tolerated due to their safety profile. Despite their successful healing rate, the incidence of reoccurrence within a year was frequently reported. This occurrence is mainly associated with accumulation of neutrophil and production of reactive oxygen species which lead to an incomplete healing process. The treatment regime is also associated with various side effects such as gynecomastia, infertility, osteoporosis, vitamin (iron, magnesium, and $\mathrm{B}_{12}$ ) deficiencies and hypergastrinemia. These symptoms can last for a long period, during and after discontinuation of the treatment. Therefore, there is an urgent need for development of novel drugs that can be administered for long-term with reduced side effects $[6,7]$.

Plant derived compounds have been used in traditional medicine since the ancient times for various ailments and diseases treatment. Now, medicinal herbs have become clinically viable for the management/treatment of gastric ulcer over synthetic drugs due to their potentially improved safety and efficacy [8]. Ocimum sanctums is used in Ayurveda and Siddha systems for asthma, cold, colic pain, cough, digestive disorders, diarrhea, headache and fever. Ocimum sanctum fresh leaves and stem extract yields an important bioactive compound, called cirsilineol. Cirsilineol is a rich phenolic antioxidant compound with various pharmacological importance such as anti-bacterial activity, anti-cancer activity, anti-diabetic activity, anti-fertility activity and anti-lipidemic activity [9-12]. Despite the extensive use of cirsilineol both traditionally and clinically, there was no data on gastroprotective mechanisms. Thus, this study was conducted to provide a scientific base for the use of cirsilineol for its anti-ulcer action against gastric ulcer.

\section{METHODS}

\section{Chemicals}

Cirsilineol of an analytical standard were purchased from Sigma-Aldrich, St. Louis, MO, USA for the present investigation. The following chemicals were acquired from Sigma-Aldrich: hydrochloric acid $(\mathrm{HCl}) /$ ethanol, sodium hydroxide $(\mathrm{NaOH})$, topfer reagent, cyanide/ferricyanide reagent, trichloroacetic acid, thiobarbituric acid, tetrahydrobenzofluorene, aprotinin, pro- inflammatory ELISA kit and haematoxylin and eosin (H\&E) staining solution. Other chemicals and solvents incorporated in this experiment were also from analytical grade.

\section{Experimental protocol}

\section{Animal model}

A total of 24 male Sprague-Dawley rats (6-8 weeks, 150-180 g) were caged under controlled laboratory environment and allowed to tap water and rodent pellets ad libitum. The acclimatization to experimental condition was performed for seven days in animal house prior to commencement of the experimentation. All further protocols were performed to minimize suffering. The experimental protocol was design according to international guidelines was approved by the Institutional Animal Ethics Committee. The ethical approval was provided by animal ethics committee of Xichang People's Hospital (approval no.: XCRMYY0910).

\section{Study design}

The rats were separately categorized into four groups and each had six animals $(\mathrm{n}=6)$. The dosage selection for cirsilineol (dissolved in $0.1 \%$ Tween 80 ) was done according to results of preliminary studies. The administration of cirsilineol was made via oral route $30 \mathrm{~min}$ before exposure to $\mathrm{HCl} /$ ethanol.

Group I: 0.1\% Tween 80 (normal saline)

Group II: $\mathrm{HCl} /$ ethanol

Group III: cirsilineol $(20 \mathrm{mg} / \mathrm{kg})+\mathrm{HCl} / \mathrm{ethanol}$

Group IV: cirsilineol (40 mg/kg) + HCl/ethanol

Prior to $24 \mathrm{~h}$ fasting, $\mathrm{HCl} / \mathrm{ethanol}(0.1 \mathrm{ml} / 20 \mathrm{~g})$ mixture $(0.15$ $\mathrm{M} \mathrm{HCl}$ in $98 \%$ ethanol) was used to induce the formation of gastric lesions in the rats through oral administration, following the method of Jin et al. [13]. Normal rats were substituted with the equal volume of $0.1 \%$ Tween 80 . Subsequently, all the rats were sacrificed after $1 \mathrm{~h}$ of sample administration. The blood sample was collected and stomach was excised with the gastric contents were aspirated before washing with normal saline for the gastroprotective assessment. All biochemical test protocols were performed following El-Maraghy et al. [14] and Yang et al. [15].

\section{Measurement of gastric ulcerative lesions}

The cleansed stomach was cut open along the great curvature, washed again with iced cold phosphate buffer (PBS) solution for assessment total ulcer area using inverted microscope with digital camera.

\section{Measurement of ulceration index}

Following, the stomach sample were subjected for the determination of gastric ulcer area $\left(\mathrm{mm}^{2}\right)$ using the image J software. The length and width of the ulcer was measured using a ruler to assess the total ulcer area. The following formula was used to obtain the percentage of ulcer inhibition (\%) by the treatment and the obtained ulcer index was expressed as mean score value for each rat:

Percentage inhibition $(\%)=$ [Ulcerated area $($ model $)$ - Ulcerated area (treated)] / Ulcerated area (model) $\times 100 \%$ 


\section{Measurement of $\mathrm{pH}$ and total acidity}

The $\mathrm{pH}$ of gastric juice content was obtained using digital $\mathrm{pH}$ meter by dipping the electrode into the beaker containing gastric juice. Thereafter, one milliliter of centrifuged gastric juice titrated against $0.1 \mathrm{~N} \mathrm{NaOH}$ using the Topfer reagent for determination of free acidity and $1 \%$ phenolphthalein in alcoholic solution for total acidity, indicated by changes in color (red to colorless to pink). The sum of both titrations was for total acidity.

\section{Measurement of hemoglobin value}

A clean and dry test tube was used to pipette out $5 \mathrm{ml}$ of buffered cyanide/ferricyanide reagent. Approximately, $0.02 \mathrm{ml}$ of blood was mixed into the tube and incubated at $30^{\circ} \mathrm{C}$. The resulting reaction mixture was subjected to absorbance reading at 546 $\mathrm{nm}$. The concentration of hemoglobin was estimated using the equation; $\mathrm{C}(\mathrm{g} / 100 \mathrm{ml})=36.77 \times$ Abs.

\section{Measurement of pro-inflammatory cytokines}

A portion of the stomach tissue was homogenized in PBS $(\mathrm{pH}$ 7.4) and centrifuged at a high speed $(10,000 \times g)$ for $20 \mathrm{~min}$ at $4^{\circ} \mathrm{C}$ using centrifuge machine. The supernatant's protein concentration was determined in the homogenate of stomach tissue samples. The tumor necrosis factor alpha (TNF- $\alpha$ ), interleukin-1 beta (IL-1 $\beta$ ) and interleukin-6 (IL-6) concentrations in were measured using commercially available ELISA kit. The homogenized supernatant yielded to a roughly of $10 \% \mathrm{w} / \mathrm{v}$ of homogenate. Then, the supernatant was further centrifuged at 1,500 rpm for $20 \mathrm{~min}$, and the recovered supernatant was used for the determination of inflammatory mediators by ELISA kits. The results obtained were expressed as cytokines concentration in lung tissue homogenates ( $\mu \mathrm{g} / \mathrm{mg}$ protein). The cytokines levels were determined by comparing to the standard graph.

\section{Measurement of lipid peroxidation activity}

The measurement of lipid peroxidation, the end product of malondialdehyde (MDA) was determined using TBARS method. Briefly, the stomach's homogenate supernatant was heated at a low $\mathrm{pH}(\mathrm{HCl})$ with $\mathrm{TBA}$ at $90^{\circ} \mathrm{C}$ for $30 \mathrm{~min}$ to produce a pink chromogen. The reaction mixture was cooled and centrifuged before subjecting to spectrophotometric absorbance at $535 \mathrm{~nm}$. An extinction coefficient of $1.56 \times 10^{5} \mathrm{M}^{-1} \mathrm{~cm}^{-1}$ was used to calculate the TBARS value for the sample. The results are expressed as lipid peroxidation (LPO) $\mu \mathrm{mol} / \mathrm{mg}$ protein.

Measurement of superoxide dismutase enzymes (SOD) activity

The SOD value in the supernatant of stomach homogenate, was determined following Marklund and Marklund [16] method. Principally, the SOD concentration was determined on its capability to increase the rate of auto-oxidation of tetrahydrobenzofluorene in the tested sample. One unit of SOD was assumed as enzyme amount of halted pyrogallol oxidation by half. The SOD activity was recorded spectrophotometrically at $525 \mathrm{~nm}$ and articulated as $\mathrm{U} / \mathrm{mg}$ protein.

\section{Measurement of catalase (CAT) activity}

CAT activity was done according to the method prescribed by Aebi [17]. The stomach homogenate was diluted with distilled water and the resulting absorbance was recorded at $240 \mathrm{~nm}$. One unit of CAT was demarcated as the enzyme amount essential to decompose $1 \mu \mathrm{mol}$ of hydrogen peroxide $\left(\mathrm{H}_{2} \mathrm{O}_{2}\right) / \mathrm{min}$. The CAT enzyme activity was expressed as $\mathrm{U} / \mathrm{mg}$ protein.

\section{Measurement of serum biochemical parameters}

The blood samples were collected in heparinized/non-heparinized syringes and placed into polystyrene micro-tubes. After clotting process, the blood was centrifugation at $4,000 \times g$ for $7 \mathrm{~min}$, to obtain the serum. The serum was separated using EDTA-treated pasteur pipettes. The remaining blood cells were washed with isotonic saline and the buffy coat was discarded and processed further for the preparation of serum biochemical analysis before storing in $-80^{\circ} \mathrm{C}$. The serum samples were analyzed using autoanalyzer to evaluate possible changes in serum biochemical parameters (liver function test, renal function test and lipid profile).

\section{Measurement of histopathological analysis}

The section of stomach sample was fixed flat on filter papers using $10 \%$ formaldehyde for $48 \mathrm{~h}$. The tissues were then processed sequentially in graded alcohol for dehydration. Further, the tissues were impregnated with paraffin wax for half an at least hour at $56^{\circ} \mathrm{C}$ and formed into a block. The blocks were sliced into $5 \mu \mathrm{m}$ by rotary microtome, dewaxed, rehydrated and stained with $\mathrm{H} \& \mathrm{E}$ stain. The slides were viewed by light microscope for histological significance.

\section{Statistical evaluation}

The analytical method was validated as mean \pm SE of at least triplicate experiments and calculated using SPSS 19.0 software (IBM Co., Armonk, NY, USA). Assessments between experimental groups were scrutinized with the Student-Newman-Keuls multiple comparison test. $\mathrm{p}<0.05$ were deliberated as statistically significant.

\section{RESULTS}

\section{Effect of cirsilineol on the gastric ulcer markers in $\mathrm{HCl} /$ ethanol induced gastric ulcer rats}

In Fig. 1, the effect of orally administered cirsilineol on $\mathrm{HCl}$ / ethanol-induced gastric lesion in rats was shown as ulcer index and ulcer markers (total acidity, $\mathrm{pH}$ and hemoglobin). The ulcer scores seen in the rats treated with cirsilineol (Group III) were significantly lower $(\mathrm{p}<0.05)$ than those in the ulcer group (II) alone. The sizes of the ulcer areas were also reduced in the rats treated with higher dose of cirsilineol (Group IV) $(\mathrm{p}<0.01)$. Similarly, the impact of cirsilineol (Group III \& IV) on gastric acidity was ameliorated compared to the ulcer group (II). The effect was normalized to the control group (I). As for the $\mathrm{pH}$ of the gastric contents, a slight increase was observed in the rats treated with cirsilineol (Group III) than the ulcer group (II). Rats treated with 

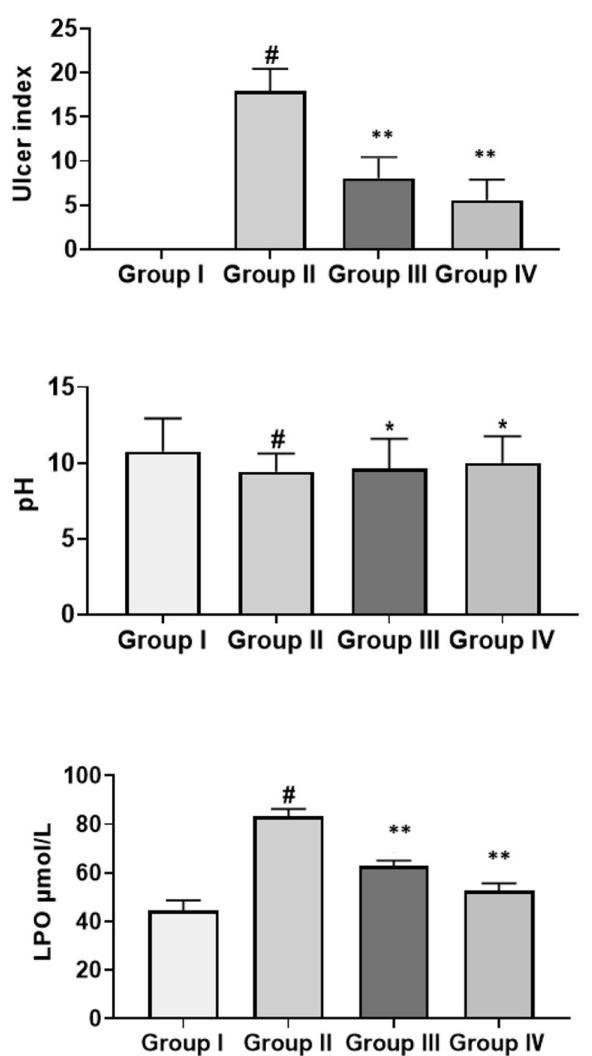

TNF-a

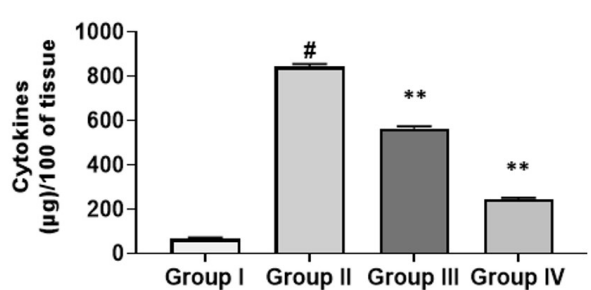

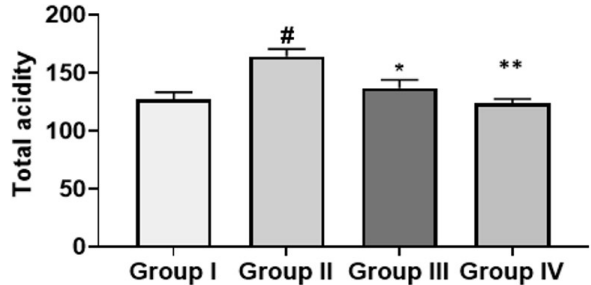

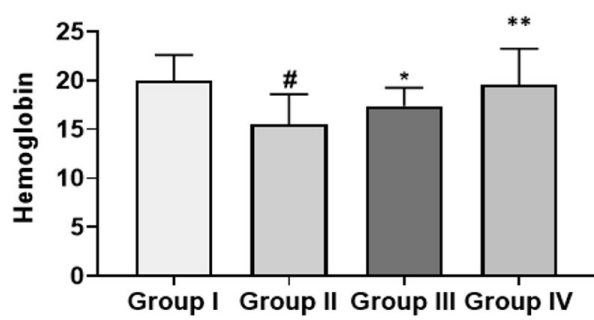

Fig. 1. The ulcer index scores and ulcer markers by cirsilineol treatment. Results were articulated as mean \pm SEM from 6 individual rats. Group I: control rats, Group II: untreated hydrochloric acid $(\mathrm{HCl}) /$ ethanol rats, Group III: cirsilineol $(20 \mathrm{mg} / \mathrm{kg})-\mathrm{HCl} /$ ethanol rats and Group IV: cirsilineol (40 mg/kg)-HCl/ ethanol rats. "Significant difference by $(p<0.05)$ from Group I, *significant difference by $(p<0.05)$ from Group II, ** significant difference by $(p<0.01)$ from Group II.
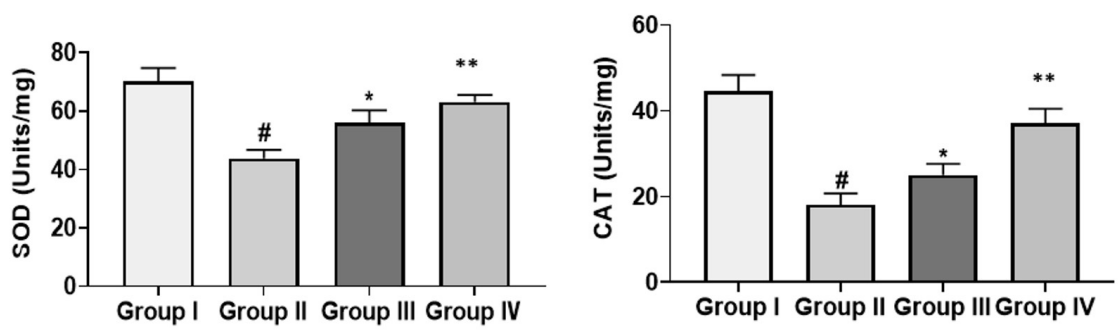

IL-1 $\beta$

IL-6
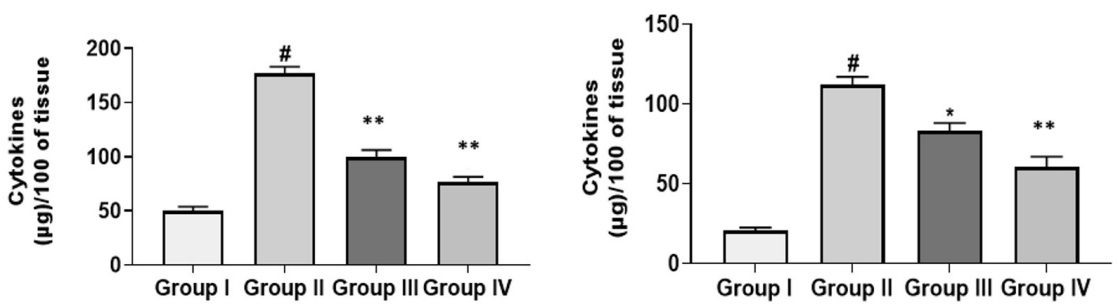

Fig. 2. The inflammatory cytokines (TNF- $\alpha$, IL-1 $\beta$, and IL-6) and anti-oxidative expression (SOD, CAT, and LPO) by cirsilineol treatment. Results were articulated as mean \pm SEM by $(p<0.05)$ from 6 individual rats. Group I: control rats, Group II: untreated hydrochloric acid $(\mathrm{HCl}) / \mathrm{ethanol}$ rats, Group III: cirsilineol (20 mg/kg)-HCl/ethanol rats and Group IV: cirsilineol $(40 \mathrm{mg} / \mathrm{kg})-\mathrm{HCl} /$ ethanol rats. TNF- $\alpha$, tumor necrosis factor alpha; IL-1 $\beta$, interleukin-1 beta; IL-6, interleukin-6; SOD, superoxide dismutase; CAT, catalase; LPO, lipid peroxidation. "Significant difference by $(p<0.05)$ from Group I, ${ }^{*}$ significant difference by $(p<0.05)$ from Group II, ** significant difference by $(p<0.01)$ from Group II.

higher dose of cirsilineol (Group IV) implied the same increase in $\mathrm{pH}$ level. A similar pattern was observed in hemoglobin level when cirsilineol was treated in rats, induced with $\mathrm{HCl} /$ ethanol.

\section{Effect of cirsilineol on the production of inflammatory mediators in $\mathrm{HCl} / \mathrm{ethanol}$ induced gastric ulcer rats}

In Fig. 2, the gastroprotective action of cirsilineol showed the involvement of inflammatory signals against $\mathrm{HCl} /$ ethanolinduced gastric lesion in rats. In ulcer group (II), the TNF- $\alpha$ expression was up regulated markedly $(\mathrm{p}<0.05)$ as well as IL-1 $\beta$, IL-6 levels than in control group (I). Treatment with cirsilineol diminished TNF- $\alpha$, IL-1 $\beta$, and IL-6 levels significantly $(\mathrm{p}<0.01)$ upon induction by $\mathrm{HCl} /$ ethanol. In other way, oral administration of cirsilineol produced a significant alteration which was normalized to the control group (I).

\section{Effect of cirsilineol on the production of anti-oxidative expression in $\mathrm{HCl} /$ ethanol induced gastric ulcer rats}

In Fig. 2, the antioxidant defense property of cirsilineol was shown by evaluating the SOD, CAT, and LPO activity against $\mathrm{HCl} /$ ethanol-induced gastric lesion in rats. The SOD and CAT activities decreased in the ulcer group (II), respectively, compared with those in the control group (I). However, in cirsilineol treated groups (III \& IV), a significant $(\mathrm{p}<0.01)$ increase in SOD and 
CAT activities were observed. On the other hand, the LPO content was significantly $(\mathrm{p}<0.05)$ higher in the gastric model group (II), compared with that in the control group (I). In cirsilineol treated groups (III \& IV), the LPO contents were lower $(\mathrm{p}<0.01)$, compared with those in the ulcer group (II).

\section{Effect of cirsilineol on the production of liver function enzymes in $\mathrm{HCl} /$ ethanol induced gastric ulcer rats}

In Fig. 3, the oral administration of cirsilineol on $\mathrm{HCl} /$ ethanolinduced gastric lesion in rats led to significant alteration in liver function parameters (alkaline phosphatases [ALP], alanine aminotransferase [ALT], and aspartate aminotransferase [AST]). The ingestion of $\mathrm{HCl} / \mathrm{ethanol}$ in group II causes a significant $(\mathrm{p}<0.05)$ elevation of ALP, ALT, and AST levels in serum compared with that of the control group (I). The administration of cirsilineol moderately prevented the elevation of serum level of ALP, ALT, and AST $(\mathrm{p}<0.05)$.

\section{Effect of cirsilineol on the production of metabolic acidosis in $\mathrm{HCl} /$ ethanol induced gastric ulcer rats}

In Fig. 4, the activity of cirsilineol on the electrochemical balance in $\mathrm{HCl} /$ ethanol-induced gastric lesion in rats were illustrated. The ulcer group (II) presented a decline in the total carbon dioxide level resulting in high anion gap $(\mathrm{p}<0.05)$ in comparison to control group (I). Following oral administration with cirsilineol, a significant $(\mathrm{p}<0.05)$ elevation in the carbon dioxide level, thus lowering the anion gap and elevation in which was almost comparable to control group (I).

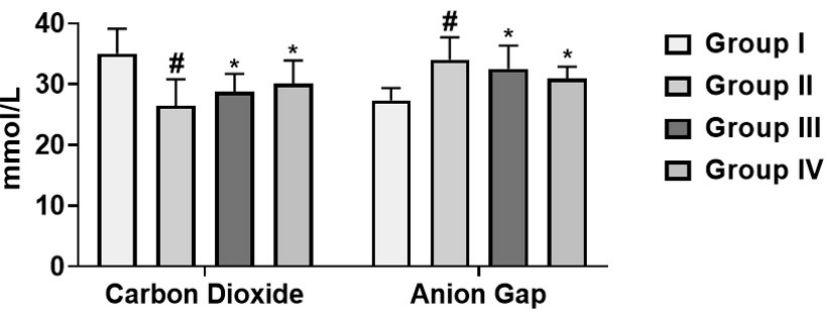

Fig. 4. The metabolic acidosis by cirsilineol treatment. Results were articulated as mean \pm SEM by $(p<0.05)$ from 6 individual rats. Group I: control rats, Group II: untreated hydrochloric acid ( $\mathrm{HCl}) /$ ethanol rats, Group III: cirsilineol (20 mg/kg)-HCl/ethanol rats and Group IV: cirsilineol $(40 \mathrm{mg} / \mathrm{kg})-\mathrm{HCl} /$ ethanol rats. "Significant difference by $(p<0.05)$ from Group I, * significant difference by $(p<0.05)$ from Group II.
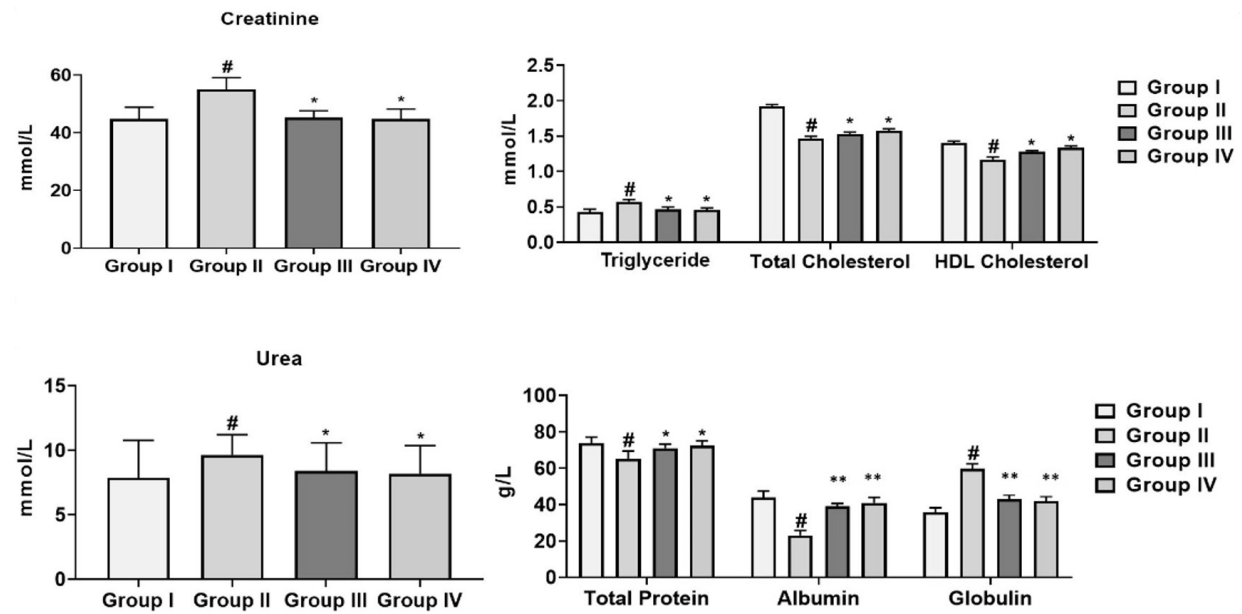

Fig. 5. The kidney function markers (urea, creatinine, total protein, albumin, and globulin) and lipid profile (triglyceride, total cholesterol, and HDL cholesterol) by cirsilineol treatment. Results were articulated as mean \pm SEM by $(p<0.05)$ from 6 individual rats. Group I: control rats, Group II: untreated hydrochloric acid (HCl)/ethanol rats, Group III: cirsilineol $(20 \mathrm{mg} / \mathrm{kg})-\mathrm{HCl} /$ ethanol rats and Group IV: cirsilineol $(40 \mathrm{mg} / \mathrm{kg})-\mathrm{HCl} /$ ethanol rats. HDL, high-density lipoprotein. " Significant difference by $(p<0.05)$ from Group I, * significant difference by $(p<0.05)$ from Group II, ** significant difference by $(p<0.01)$ from Group II. 


\section{Effect of cirsilineol on the production of kidney function markers in $\mathrm{HCl} /$ ethanol induced gastric ulcer rats}

In Fig. 5, the oral administration of cirsilineol on $\mathrm{HCl} /$ ethanolinduced gastric lesion in rats resulted in a significant alteration of kidney function parameters (urea, creatinine, total protein, albumin and globulin). The oral administration of cirsilineol in $\mathrm{HCl} /$ ethanol (groups III \& IV) causes a significant $(\mathrm{p}<0.05)$ ameliorative effect of kidney markers compared with that of the ulcer group (II). The administration of cirsilineol were normalized to normal control group (I).

\section{Effect of cirsilineol on the production of lipid markers in $\mathrm{HCl} /$ ethanol induced gastric ulcer rats}

In Fig. 5, the triglyceride, high-density lipoprotein (HDL) cholesterol and total cholesterol activity of oral administration of cirsilineol against $\mathrm{HCl} /$ ethanol-induced gastric lesion in rats are presented. The figures reveal that the administration of cirsilineol (groups III \& IV) greatly reduces $(\mathrm{p}<0.05)$ the concentration of triglyceride when compared with the ulcer group (II). However, the administration of cirsilineol (groups III \& IV) increases the concentration levels of total and HDL of cholesterol level with a significant difference when compared with the ulcer group (II) (p $<0.05)$.

\section{Effect of cirsilineol on the production of histological evidence in $\mathrm{HCl} /$ ethanol induced gastric ulcer rats}

In Fig. 6, the histological alteration in orally administrated cirsilineol against $\mathrm{HCl} /$ ethanol-induced gastric lesion in rats are illustrated. The figures reveal that the induction of $\mathrm{HCl} /$ ethanol induced gastric lesion, which was evidenced by congestion of inflammatory cell, loss of mucous membrane and necrosis. The administration of cirsilineol (group III) greatly reduces the loss of mucous membrane and inflammatory cells when compared with the ulcer group (II). The effect of cirsilineol was almost normalized to the control group (I). In addition, the histological analysis of gastric mucosa in stomach specimen treated with higher dose of cirsilineol (group IV) showed comparable patterns as cirsilineol treated group III, suggesting the treatment with cirsilineol at lower dose is sufficient to prevented $\mathrm{HCl} /$ ethanol induced gastric injury in rats.

\section{DISCUSSION}

The ideal way to resolve stomach complications are possibly to find ways to restore gastric homeostasis with certain medication which resembles dietary intakes to address their symptoms. Herein, this investigation evaluates the gastro-protective effect of the cirsilineol against $\mathrm{HCl} /$ ethanol-induced gastric injury in rats. $\mathrm{HCl} /$ ethanol-induced gastric ulcer were utilized in this study as

\section{Group I}
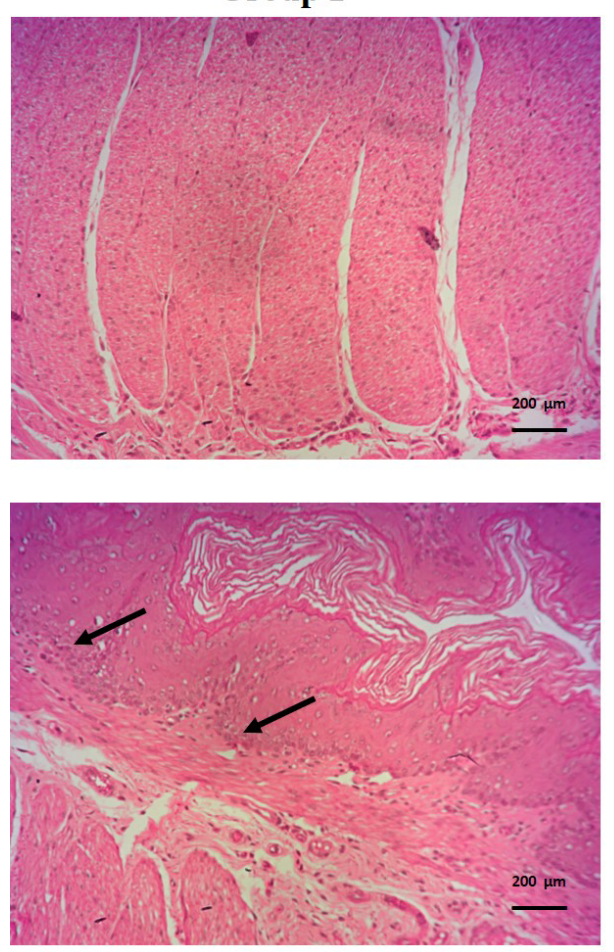

Group III
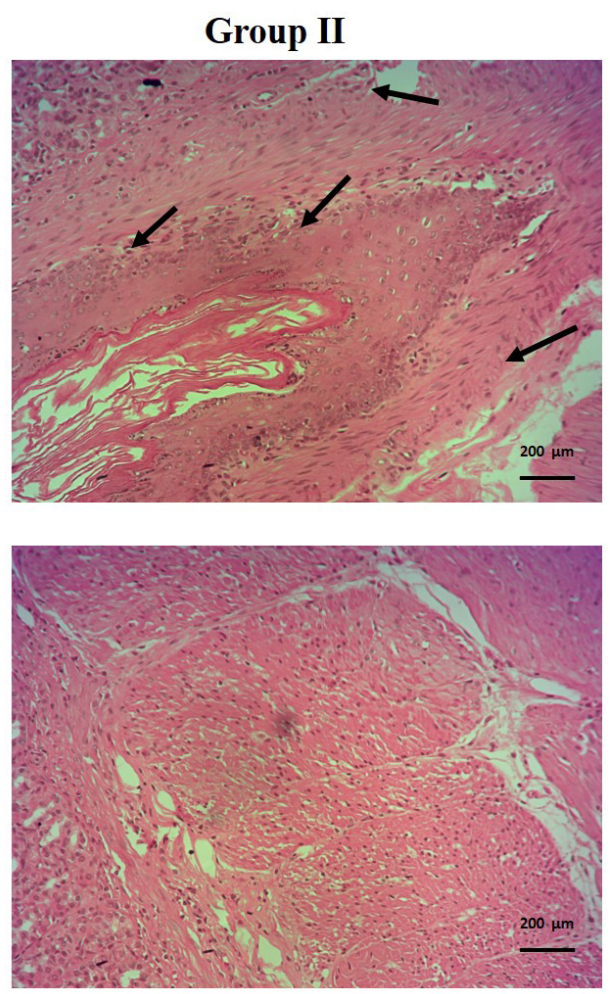

Group IV
Fig. 6. The histopathological evidence by cirsilineol treatment. Group I: control rats; Group Il: hydrochloric acid $(\mathrm{HCl})$ / ethanol model rats (black arrows indicate the inflammatory cells, submucosal and mucosal damage, necrosis); Group III: cirsilineol (20 mg/kg)-HCl/ethanol rats (black arrows indicate the inflammatory cells, mild mucosal damage) and Group IV: cirsilineol $(40 \mathrm{mg} / \mathrm{kg})-\mathrm{HCl} /$ ethanol rats. The stain method is H\&E (magnification at $\times 100$ ). 
an experimental model to investigate the protective action of cirsilineol extracted from Ocimum sanctum. This model was preferred as it is closely features the development of ulcers in humans [18]. In this model, $\mathrm{HCl}$ induce damage in the lining of stomach mucosa, while ethanol causes severe lesion by suppressing the production of mucus and enzymes. Subsequently, these creates formation of oxidative stress which changes in the permeability of gastric mucosa to produce hemorrhagic lesions, mucosal friability, extensive sub mucosal edema, inflammatory cells infiltration, and epithelial cell loss in the stomach, which are distinctive features of injury [19].

In the marker accessing assay, oral administration of $\mathrm{HCl} /$ ethanol induced gastric ulcer in rats promoted gastric ulcer area compared to the control group. Combination of $\mathrm{HCl} /$ ethanol are considered as a 'gastro-toxic' agent that causes gastric mucus diminution that considerably injures the cell membrane of stomach [20]. Following oral administration with cirsilineol, the gastric lesions were significantly reduced. Possibly cirsilineol are effective against detrimental effect of $\mathrm{HCl} /$ ethanol, which could hold a gastric mucosal membrane protective activity. For these reasons, a further validation was carried out to examine the effect of cirsilineol in the role of gastro-protection. The observations showed protective indexes which supported by the percentage of lesion inhibition caused by cirsilineol in $\mathrm{HCl} /$ ethanol induced gastric ulcer rats. These results are in parallel with previous work which evaluated the gastro-protection of various synthetic compounds and authenticate the gastro-protective role of gallic acid against ethanol-induced gastric ulcer in rats [21].

In the present study, an increase in total acidity and hemoglobin concentration while decrease in $\mathrm{pH}$ were observed in $\mathrm{HCl}$ / ethanol induced gastric ulcer rats. The degree of gastric ulceration has been associated with increase in the acidity and hemoglobin activity. In addition, gastritis can root to mal-absorption of iron because gastric acid is required for the iron absorption which often results in hemoglobin deficiency disorder such as anemia [22]. Treatment with cirsilineol was found to improve the $\mathrm{pH}$ level, which shows the degree of acidity reduction. Hemoglobin level was also found to be enhanced in cirsilineol animals. These modulating activity are highly anticipated for gastro-protective effect of an anti-ulcer agent. This anti-ulcerogenic effect were in agreement with a previous study conducted by Mahendran et al. [23] using Garcinia cambogia extract which amend the hemoglobin, volume and total acidity level of gastric juice in ulcerated rats.

The following study proved that $\mathrm{HCl} /$ ethanol increased the gastric level of inflammatory cytokines, TNF- $\alpha$, IL-1 $\beta$, and IL- 6 , while cirsilineol, modulated the levels of TNF- $\alpha$, IL-1 $1 \beta$, and IL6. In gastric ulcer, $\mathrm{HCl} /$ ethanol causes inflammation to initiates macrophages release and inflammatory cytokines (TNF- $\alpha$, IL-1 $\beta$, and IL-6) to promote neutrophil accumulation at the target site for instigation of mucosal barrier destruction [24]. This data was in a line with a study conducted on gallic acid against ethanolinduced gastric ulcer rats which shows its anti-inflammatory effect by inhibiting the levels of TNF- $\alpha$ and IL-6 elevation [25]. Pro-inflammatory cytokine TNF- $\alpha$ activates the NF-k $\beta$ pathway, triggering the transcription for macrophages and cytokines to mediate inflammation. Mechanism of cirsilineol against $\mathrm{HCl}$ / ethanol-induced gastric lesion was apparent from the results of reduced inflammatory cytokines, which automatically inhibits the activation of NF-k $\beta$ pathway. Oral administration of cirsilineol might have restored the healing mechanism by re-epithelialization of mucosal and submucosal of the stomach cell membrane in $\mathrm{HCl} /$ ethanol induced gastric ulcer rats. Restoration of mucosal layer is considered the first indication of healing process against gastric ulcer damage [26]. This mechanism was exhibited by cirsilineol that could also be a key factor in the prevention of gastric ulcer.

The ELISA assay corroborated that oral administration of $\mathrm{HCl} /$ ethanol causes severe oxidative stress in stomach tissue as witnessed in control group by significant increase in the level of lipid peroxidation (MDA) which concurrently inhibited of the activity of antioxidant enzymes level, CAT and SOD. Manifestation of oxidative stress prompts abnormal level of free radicals which depletes the concentration of antioxidant enzymes, which function as defense mechanism against damaging free radicals [27]. The administration of cirsilineol improves the cellular antioxidant defenses by elevating the CAT and SOD while decreasing the level of lipid peroxidation. More recently, a study by Kumari and Anbarusa [28] reported similar protective activity by C-phycocyanin against selenite cataract rat model, by its ability to scavenge the free radicals. Similarly, cirsilineol conserved the antioxidant activity (SOD and CAT), specifying its antioxidant potential, thus protecting the gastric mucosa against lipid peroxidation damage. This data further confirms that cirsilineol has gastro-protective properties against the development of $\mathrm{HCl} /$ ethanol ulcers in rats. The lipid profile (total cholesterol, HDL, triglyceride levels) is associated with oxidative stress since increase of triglyceride level in gastric model group can be related to the excessive formation of lipid peroxides. Cirsilineol was able to reduce triglyceride levels in a dose-dependent manner by increasing the antioxidant effect, suppressing the production of lipid peroxides. Moreover, the total cholesterol and HDL levels have slightly reversed towards normal upon administration of cirsilineol due to the reversal of oxidative stress condition.

Finally, the toxicity profile of cirsilineol was investigated by accessing the serum biochemical parameters of liver, kidney and lipid function markers. Upon exposure to toxic condition such as $\mathrm{HCl} /$ ethanol or drugs, liver is impaired where by the ALP, ALT, and AST are excessively secreted into bloodstream. Another indication of liver injury is the release of plasma proteins such as total protein, albumin, and globulin [29]. These are estimation of excretory and metabolic estimation of liver where the levels were suppressed is a signal of chronic liver damage. As for renal damage, biochemical indicators are serum urea and creatinine which are excreted out of the body. However, during renal failure, the 
excretion functions are impaired. In addition, the level of blood cholesterol is implicative of metabolic problem in the liver [30]. Here, ameliorative effect of cirsilineol was observed in several markers of liver and kidney against $\mathrm{HCl}$ /ethanol-induced gastric lesions in rats. This was evidently justified with histopathological analysis revealing reduced damage in the stomach ulcer suggesting the cirsilineol anti-ulcer activity against experimentally induced gastric ulcer. These results give a further insight into the effect of cirsilineol on the organs of the rats for the safety claim of the cirsilineol.

Overall, the obtained results suggest cirsilineol employed an appreciable anti-ulcerative effect against $\mathrm{HCl}$ /ethanol-induced gastric lesions in rats. This activity can be associated with the improvement of antioxidant defense system to attenuate the inflammatory response for the improvement of the lesion accountable for the development of gastric ulcers. In addition, improvement in the liver, kidney and lipid parameter displayed a non-toxic profile of cirsilineol, clinically. However, a further comprehensive study is required to determine the mechanisms responsible for the gastro-protective action exhibited by cirsilineol.

\section{CONFLICTS OF INTEREST}

The authors declare no conflicts of interest.

\section{REFERENCES}

1. Malfertheiner P, Chan FK, McColl KE. Peptic ulcer disease. Lancet. 2009;374:1449-1461.

2. Hamedi S, Arian AA, Farzaei MH. Gastroprotective effect of aqueous stem bark extract of Ziziphus jujuba L. against $\mathrm{HCl}$ / Ethanol-induced gastric mucosal injury in rats. J Tradit Chin Med. 2015;35:666-670.

3. Sharifi-Rad M, Fokou PVT, Sharopov F, Martorell M, Ademiluyi AO, Rajkovic J, Salehi B, Martins N, Iriti M, Sharifi-Rad J. Antiulcer agents: from plant extracts to phytochemicals in healing promotion. Molecules. 2018;23:1751.

4. Lin KJ, García Rodríguez LA, Hernández-Díaz S. Systematic review of peptic ulcer disease incidence rates: do studies without validation provide reliable estimates? Pharmacoepidemiol Drug Saf. 2011;20:718-728.

5. Wang AY, Peura DA. The prevalence and incidence of Helicobacter pylori-associated peptic ulcer disease and upper gastrointestinal bleeding throughout the world. Gastrointest Endosc Clin N Am. 2011;21:613-635.

6. Kangwan N, Park JM, Kim EH, Hahm KB. Quality of healing of gastric ulcers: natural products beyond acid suppression. World J Gastrointest Pathophysiol. 2014;5:40-47.

7. Dacha S, Razvi M, Massaad J, Cai Q, Wehbi M. Hypergastrinemia. Gastroenterol Rep (Oxf). 2015;3:201-208.

8. Raza H, Abbas Q, Hassan M, Eo SH, Ashraf Z, Kim D, Phull AR, Kim SJ, Kang SK, Seo SY. Isolation, characterization, and in silico, in vitro and in vivo antiulcer studies of isoimperatorin crystallized from Ostericum koreanum. Pharm Biol. 2017;55:218-226.

9. Mishra P, Mishra S. Study of antibacterial activity of Ocimum sanctum extract against gram positive and gram negative bacteria. Am J Food Technol. 2011;6:336-341.

10. Mondal S, Mahapatra SC, Mirdha BR, Naik SN. Antimicrobial activities of essential oils obtained from fresh and dried leaves of Ocimum sanctum (L.) against enteric bacteria and yeast. Acta Horticult. 2007;756:267-269.

11. Patil R, Patil R, Ahirwar B, Ahirwar D. Isolation and characterization of anti-diabetic component (bioactivity-guided fractionation) from Ocimum sanctum L. (Lamiaceae) aerial part. Asian Pac J Trop Med. 2011;4:278-282.

12. Nagarajun S, Jain HC and Aulakh GS. Indigenous plants used in fertility control. In: Atal CK, Kapoor BM, editors. Cultivation and utilization of medicinal plants. PID CSIR; 1989. p.558.

13. Jin X, Liu Q, Dang M, Zhang W, Li X. Gastroprotective and ulcer healing properties of ethanol extract of Alpinia conchigera rhizome in experimentally induced gastric ulcer in rats. Trop J Pharm Res. 2019;18:1873-1879.

14. El-Maraghy SA, Rizk SM, Shahin NN. Gastroprotective effect of crocin in ethanol-induced gastric injury in rats. Chem Biol Interact. 2015;229:26-35.

15. Yang Y, Yin B, Lv L, Wang Z, He J, Chen Z, Wen X, Zhang Y, Sun W, Li Y, Zhao Y. Gastroprotective effect of aucubin against ethanolinduced gastric mucosal injury in mice. Life Sci. 2017;189:44-51.

16. Marklund S, Marklund G. Involvement of the superoxide anion radical in the autoxidation of pyrogallol and a convenient assay for superoxide dismutase. Eur J Biochem. 1974;47:469-474.

17. Aebi HE. Catalase. In: Bergmeyer HU, editor. Methods of enzymatic analysis. Weinhem: Verlag Chemie; 1983. p.273-286.

18. da Silva LM, Boeing T, Somensi LB, Cury BJ, Steimbach VM, Silveria AC, Niero R, Cechinel Filho V, Santin JR, de Andrade SF. Evidence of gastric ulcer healing activity of Maytenus robusta Reissek: in vitro and in vivo studies. J Ethnopharmacol. 2015;175:75-85.

19. Jelski W, Kozlowski M, Laudanski J, Niklinski J, Szmitkowski M. The activity of class I, II, III, and IV alcohol dehydrogenase (ADH) isoenzymes and aldehyde dehydrogenase (ALDH) in esophageal cancer. Dig Dis Sci. 2009;54:725-730.

20. Zhou D, Yang Q, Tian T, Chang Y, Li Y, Duan LR, Li H, Wang SW. Gastroprotective effect of gallic acid against ethanol-induced gastric ulcer in rats: involvement of the Nrf2/HO-1 signaling and antiapoptosis role. Biomed Pharmacother. 2020;126:110075.

21. Arab HH, Salama SA, Omar HA, Arafa el-SA, Maghrabi IA. Diosmin protects against ethanol-induced gastric injury in rats: novel anti-ulcer actions. PLoS One. 2015;10:e122417.

22. Saranya P, Geetha A, Selvamathy SM. A biochemical study on the gastroprotective effect of andrographolide in rats induced with gastric ulcer. Indian J Pharm Sci. 2011;73:550-557.

23. Mahendran P, Sabitha KE, Devi CS. Prevention of HCl-ethanol induced gastric mucosal injury in rats by Garcinia cambogia extract and its possible mechanism of action. Indian J Exp Biol. 2002;40:5862.

24. Abdelwahab SI. Protective mechanism of gallic acid and its novel derivative against ethanol-induced gastric ulcerogenesis: involvement of immunomodulation markers, Hsp70 and Bcl-2-associated X protein. Int Immunopharmacol. 2013;16:296-305. 
25. Song SH, Kim JE, Sung JE, Lee HA, Yun WB, Lee YH, Song H, Hwang D. Anti-ulcer effect of Gallarhois extract with anti-oxidant activity in an ICR model of ethanol/hydrochloride acid-induced gastric injury. J Tradit Complement Med. 2018;9:372-382.

26. Swarnakar S, Mishra A, Ganguly K, Sharma AV. Matrix metalloproteinase- 9 activity and expression is reduced by melatonin during prevention of ethanol-induced gastric ulcer in mice. J Pineal Res. 2007;43:56-64.

27. Antonisamy P, Duraipandiyan V, Aravinthan A, Al-Dhabi NA, Ignacimuthu S, Choi KC, Kim JH. Protective effects of friedelin isolated from Azima tetracantha Lam. against ethanol-induced gastric ulcer in rats and possible underlying mechanisms. Eur J Pharmacol. 2015;750:167-175.
28. Kumari RP, Anbarasu K. Protective role of C-phycocyanin against secondary changes during sodium selenite mediated cataractogenesis. Nat Prod Bioprospect. 2014;4:81-89.

29. Sidahmed HM, Hashim NM, Mohan S, Abdelwahab SI, Taha MM, Dehghan F, Yahayu M, Ee GC, Loke MF, Vadivelu J. Evidence of the gastroprotective and anti- Helicobacter pylori activities of $\beta$-mangostin isolated from Cratoxylum arborescens (vahl) blume. Drug Des Devel Ther. 2016;10:297-313.

30. Al-Wajeeh NS, Hajrezaie M, Al-Henhena N, Kamran S, Bagheri E, Zahedifard M, Saremi K, Noor SM, Ali HM, Abdulla MA. The antiulcer effect of Cibotium barometz leaves in rats with experimentally induced acute gastric ulcer. Drug Des Devel Ther. 2017;11:995-1009. 